\title{
Computer-Based Determination of the Newborn's Femoral Head Coverage Using Three-Dimensional Ultrasound Scans
}

\author{
Heinrich M. Overhoff ${ }^{1}$, Djordje Lazovic ${ }^{2}$, Ute von Jan ${ }^{1}$, Peter Heinze \\ ${ }^{1}$ University of Hildesheim, Department for Computer Science, Postfach 1013 63, \\ D-31113 Hildesheim, Germany \\ martin@med-informatik.uni-hildesheim.de \\ ${ }^{2}$ Hannover Medical School, Orthopedic Department, Heimchenstraße 1 - 7 , \\ D-30625 Hannover, Germany \\ lazovic@t-online.de
}

\begin{abstract}
An automatic image analysis method is presented, which finds the diagnostic landmarks for the determination of the femoral head coverage according to Graf's method in a 3-D ultrasound image volume. Some of the process steps depend on tuneable parameters. It is a typical experience that the quality of images differs between investigators and patients respectively. An image analysis algorithm therefore should produce results which are robust against such varying image quality. The sensitivity of intermediary and final image analysis process steps versus expert defined parameters is investigated. This analysis are performed in a pilot study on 3-D image data of 10 newborns.
\end{abstract}

\section{Introduction}

Hip joint dysplasia is the most frequent congenital skeletal disease [1], with exemplary prevalences in newborns are about $2 \%$ in the USA and the UK, $4 \%$ in Central Europe, and $10 \%$ in Japan. In the 1980's Graf developed a diagnostic technique, which is meanwhile established as standard method for the investigation and quantification of the newborn's hip joint findings in Central Europe. Graf's technique is based on a 2-D ultrasound image taken in the so-called standard plane [2], [3], [4]. It focuses on the analysis of the relative location of the newborn's femoral head and acetabulum. The femoral head coverage is then quantified by geometric measures taken from the 2-D image. This method leads to standardised and comparable findings, but the imaging and inspection of the hip joint demands high skills. It has to be claimed that the hip joint of the newborn usually is not ossified. Therefore, the hip joint itself is invisible in the ultrasound images: the femoral head of the newborn is cartilaginous, and the same holds for the non-ossified junction of iliac, ischial and pubic bone, the so called hypsiloid cartilage. These structures can only be identified indirectly by bounding landmarks. Another disadvantage is the systematic reduction 
of a spatial geometry to a single or a few 2-D plane cuts. Thus, findings visible in non-standardised image planes must be interpreted and documented individually, which reduces the comparability of clinical reports.

The use of 3-D ultrasound for several diagnostic investigations has been studied in the past few years including the visualisation of the newborn's hip joint [5], [6]. The reporting of 3-D ultrasound image data sets primarily aims on a more precise inspection. An automatic processing of Graf's diagnostic method or a quantification of the femoral head coverage, e.g. the derivation of geometric measures of the joint based on a spatial representation, to our knowledge has not been reported so far (cf. [7]).

In this study, an automatic image analysis method is presented, which finds the diagnostic landmarks for the determination of the femoral head coverage according to Graf's method in a 3-D ultrasound image volume. To ensure the robustness of the image analysis algorithm, the sensitivity of intermediary and final image analysis process steps versus central process parameters was investigated. This analysis are performed in a pilot study on 3-D image data of 10 newborns. Final results were taken for a robust parametrization.

\section{Materials and Methods}

For data sets of 10 newborns ( 2 days - 12 weeks) an image analysis was performed automatically. In this application certain structures with typical shapes are expected in the images. Therefore the automatic image analysis is organised in two steps. First a data-driven image pre-processing is performed, and their results are investigated in a second step using mathematical functions which model the expected forms. From a methodical point of view the sensitivity of model-based image processing vs. the preprocessed raw-data is investigated. Implicitly the assumption is made that data-driven image processing tends to be error sensitive, while model-based approaches are more robust. Therefore only the robustness of the image analysis vs. the pre-processing steps is analysed. Thus the location of resulting landmarks and sphere parameters is investigated vs. pre-processing parameters.

\subsection{Image processing}

Image acquisition. Images of the newborn's hip are taken during a free-hand sweep around the coronal plane. A typical image data set includes about 30 images of about 200 pixel $\times 200$ pixel size. A conventional ultrasound imaging system (Kontron AI 5200 ) with a $7.5 \mathrm{MHz}$-Linear Array transducer is used. The pixel's edge measures are about $0.23 \mathrm{~mm} \times 0.23 \mathrm{~mm}$. The image contrast is manually controlled by a depthdependent signal gain.

For the determination of the transducer motion a specially developed sensing system is used. By the parallel orientation of transducer and image, the spatial image positions can directly be determined from the transducer movement measurements. 
The sensing system is optimised for rotation measurements, its accuracy is $\leq 0.1^{\circ}$. During the image data collection, the scan head is only rotated around two axes.

The data are acquired by an IBM-PC. The images are provided by the video output of the US system and digitised with a video grabbing card. The image processing, the automatic determination of a sphere approximating the femoral head, and the identification of the acetabular landmarks were performed on an IBM-compatible PC (166 MHz, 64 MBytes RAM).

Transducer kinematics. The motion of the transducer is described according to the kinematics of masspoints known from mechanics [8]. As the transducer's object coordinate system only rotates in the world co-ordinate system, a spherical co-ordinate system is adequate to express the relative motion parameterized by the two angles 6 and $\varphi$. With these data the spatial location of the image pixels can be calculated.

Image analysis. The interpretation of a hip joint dysplasia using 2-D standard plane images is based on ultrasound reflecting structures, which have a fix relation to the hip joint. These landmarks must be identified to derive coverage measures. According to Graf's diagnostic method, these landmarks are the contour of the iliac bone as reference axis (1), the osseous edge of the acetabulum (2), the most distal osseous edge of the ilium (3), the cartilaginous acetabular labrum (4), and the reflection of the capsule (5); the numbers refer to Fig. 1. The image analysis itself is dissected into three major steps:

1. identification of the iliac bone and a region of interest, where the femoral head is suspected,

2. estimation of a sphere approximating the femoral head and determination of the $f$-angle, which is a criterion for the cartilaginous head coverage, and

3. calculation of the iliac part of the already ossified acetabulum and determination of the $\alpha$-angle, which is an indicator for the quality of the head coverage (Fig. 2).

An automatic image analysis for the femoral head approximation [7] was extended by an algorithm identifying the acetabular landmarks (2) and (3). The entire procedure is described in the following. For each process step their detail results and major process parameters are listed. The sensitivity of the image analysis results vs. these parameters is investigated to find optimal values for a robust image processing.

Formally, some notations for images are introduced. An image itself is noted as a $N_{\text {line }} \times N_{\text {row }}$-matrix $\mathbf{P}=[p(i, j)], 1 \leq i \leq N_{\text {line }}, 1 \leq j \leq N_{\text {row }}$, having grey-values $g_{\min } \leq g \leq g_{\text {MAX }}$ (here: $g_{\min }=0, g_{\text {MAX }}=255$ ). A histogram is given by $h(g)=\operatorname{card}(p(i, j)=g)$, and $H(g)$ denotes cumulated grey-value frequencies normalised by $N_{\text {line }} \cdot N_{\text {row }}$.

Determination of the region of interest for the femoral head and acetabulum. To detect the echo of the iliac bone, the image volume is binarized using a threshold $g_{\text {THRES SKELETON }}$. A skeletonization is then performed on each plane of the binarized volume, and all skeletons except the medial-most one on the proximal side of each image are discarded. These skeletons coincide with the iliac bone and are ideal- 
ised as proximal-distal lines. The distal end points of these lines serve to approximate the position of the most distal osseous edge of the ilium.

The cumulative frequency $H\left(g_{\text {THRES,SKELETON }}\right)$ is the major parameter.

Detection of the femoral head approximating sphere and the $p$-angle. At the most distal osseous edge of the ilium, a region of interest (ROI) in the form of a mirrored " $\mathrm{C}$ " is introduced. The diameter-range of the " $\mathrm{C}$ " was chosen to $20 \leq \mathrm{D} /$ pixel $\leq 60$, as $D \approx 40$ pixel is a typical femoral head radius (cf. [7]). Its centre is taken as provisional centre of the femoral head approximating sphere. A gradient image is constructed in the ROI, where the gradient's directions are determined from the provisorial centre. The cumulated grey-value frequencies $H(g)$ are calculated for the gradient image, which then is binarized using a threshold $g_{\text {THRES,CONTOUR }}$. The remaining pixels contour the only implicit visible femoral head circle. Pixels in the ROI which are located most to the provisorial centre and have restricted relative angle positions to this point are taken to construct a four-dimensional Hough-accumulator for sphere surfaces. This accumulator then is normalised by the sphere surfaces, cluster analysed and finally investigated for the most frequent sphere parameter combination. For details cf. [7].

The result of the sphere detection is a parameter vector $\left\lfloor x_{\text {centre }} y_{\text {centre }} z_{\text {centre }} D\right\rfloor$. To ease its interpretation, intersections of this sphere with each original image, i.e. circles, are visualised on the computer screen. Fig. 3 additionally shows the intersection with the expert placed reference sphere.

The cumulative frequency $H\left(g_{\text {THRES }, C O N T O U R}\right)$ is considered to be the major parameter.

Acetabulum lines and $\alpha$-angle. From anatomy the special geometric structure of the acetabular part of the iliac bone is known. The voxels bounding the femoral head in the acetabular region construct a ROI, where the osseous edge of the acetabulum and the most distal osseous edge of the ilium are found. Here only those pixels are used, which are brighter then the grey-value $H\left(g_{T H R E S, A C E}\right)$. The result of this processing step are the spatial co-ordinates of the two landmarks necessary for the calculation of Graf's $\alpha$-angle by the use of the most distal osseous edge of the ilium.

\subsection{Verification}

The results of the image analysis were tested for correctness and robustness vs. the image processing parameters. This analysis was performed to gain stable reliable results independent of image contrast. An objective determination of the parameters of the coverage parameters was not possible, as none of the children was treated surgically. Therefore, to control the automatically determined landmarks and measures, the placement of the distal iliac crest points was compared to pixels determined by three experienced experts. The same was done for the most distal edge of the ilium and the acetabular edge. To control the automatically determined femoral head approximating sphere, an expert placed virtual spheres in a 3D-scene and parametrised 
their origin and diameter. For control the original image slices intersected the virtual sphere. The section figure in each image is a circle. These circles were inserted into the images and controlled for their location. The location of the virtual femoral head sphere was corrected until this control yielded a plausible result.

The analysis of the first two process steps, defining the hip joint ROI and determination of a femoral head approximating sphere, were statistically analysed even for additional data sets [9]. Due to the smaller number of cases in this study the results of the acetabular landmarks identification was only described statistically.

\section{Results}

We could obtain data from 10 hip joints and evaluated them for this study. The mean processing time was about 8 minutes for an entire image data set.

The measurement errors for the co-ordinates of the most distal point of the iliac crest line ranged between -1 and 33 pixels for $0.7 \leq H\left(g_{\text {THRES,SKELETON }}\right) \leq 0.95$ and -1 and 10 for $0.8 \leq H\left(g_{\text {THRES.SKELETON }}\right) \leq 0.95$ joints. A statistical analysis ( $\mathrm{t}$ test) over 20 hip joints did not reject the null-hypothesis of a mean measurement error of 0 pixels $(\alpha=5 \%)$. The parameters of the approximating spheres ranged between 4 and 3 pixels for $0.7 \leq H\left(g_{\text {THRES }, C O N T O U R}\right) \leq 0.95$. Significantly $(\alpha=5 \%)$ the sphere centre's $y$-and $z$-co-ordinate and the sphere diameter measurement errors were between -2 and 2 pixels. As example for the robustness the displacement of the proximal-distal $y$-co-ordinate of the distal-most point of the pelvic crest line is presented in Fig. 4 (cf. [9]). The figure underlines the correct choice of the thresholds in [7], as for the ranges $0.8 \leq H\left(g_{\text {THRES.SKELETON }}\right) \leq 0.95$ and $0.85 \leq H\left(g_{\text {THRES CONTOUR }}\right) \leq 0.95$ significantly high correctness can be gained.

The automatically determined and the expert defined co-ordinates for the acetabular edge and the most distal edge of the ilium yielded displacements ranging from -5 to 3 pixels for all co-ordinates independently of reasonably chosen parameters $H\left(g_{\text {THRES }, A C E}\right)$.

The median errors for the co-ordinates ranged between -1 and 1 pixel $(-0.23 \mathrm{~mm}$ resp. $0.23 \mathrm{~mm}$ ) in a detail image of about 20 pixels $\times 20$ pixels size, and their $75 \%$ quantiles were 0 to 3 pixels $(0.23 \mathrm{~mm}$ resp. $0.0 \mathrm{~mm})$. We found a systematic underestimated $y$-co-ordinate of the acetabular edge.

\section{Discussion}

The presented automatic analysis of a 3-D US image volume is an attractive supplement to the conventional 2-D investigation according to Graf's method. It could be shown that the image pre-processing bases on robust methods and yields accurate results for the essential intermediary process parameters. These are the most distal osseous edge of the ilium, which serves to locate a ROI, in which the hip joint is expected, a femoral head approximating sphere, and the boundaries of the iliac bone in 
the proximal acetabulum. By the statistical analysis it turned out that the process parameter choices for the ROI-definition of the expected joint location and the determination of the approximating sphere's parameters in [7] were reasonable. The determined parameters were accurate, only the centre of the sphere tends be located imprecisely in the transducer main sweep direction.

To rate the measurement errors of the acetabular landmarks it should be mentioned that in the investigation the median of the acetabular index is $\alpha=63^{\circ}$. Assuming measurement errors with median deviations of the co-ordinates the angles are misinterpreted by $\Delta \alpha_{\text {median }}=5.3^{\circ}$, and for $75 \%$-quantile measurement deviations by $\Delta \alpha_{75 \%}$-quantile $=7.1^{\circ}$. These error measures exemplary can be related to the correctness of landmark identification an experienced physician can reach in clinical routine diagnostics of about 4 pixels for the most distal osseous edge of the ilium [10]. At the moment an interpretation of the systematic measurement error of the $y$-co-ordinate of the acetabular edge is not possible. More data sets must be investigated to analyse its origin.

As further work, robust statistical methods will be used to evaluate the femoral head parameters due to the inaccurate centre location orthogonal to the image planes. Furthermore, the geometric results should be controlled in a cadaver study to obtain objective measures.

\section{References}

1. Tönnis, D.: Die angeborene Hüftdysplasie und Hüftluxation im Kindes- und Erwachsenenalter, Springer-Verlag, Berlin Heidelberg New York (1984)

2. Exner, G.U., Mieth, G.: Sonographische Hüftdysplasie beim Neugeorenen, Schweiz Med Wochenschr (1987) 1015-1020

3. Graf, R.: The Diagnosis of congenital hip joint dislocation by the ultrasonic compound treatment. Arch Orthop Trauma Surg (1980) 117-133

4. Graf, R.: New possibilities for the diagnosis of congenital hip joint dislocation by ultrasonic compound treatment. J Ped Orthop (1983) 354

5. Graf, R., Lercher, K.: Erfahrungen mit einem 3-D-Sonographiesystem am Säuglingshüftgelenk. Ultraschall Med (1996) 218-226

6. Gerscovich, E.O., Greenspan, A., Cronan, M., Karol, L., McGahan, J.P. : Threedimensional Sonographic Evaluation of Developmental Dysplasia of the Hip: Preliminary Findings. Radiology (1994) 407-410

7. Overhoff, H.M., Lazovic, D., Franke, J., Jan, U. von: Automatic Determination of the Newborn's Femoral Head from three-dimensional Ultrasound Image Data. In: Lecture Notes in Computer Science, Vol. 1205 Springer-Verlag, Berlin Heidelberg New York (1997) 547556

8. Wittenburg, J.: Dynamics of Systems of Rigid Bodies, Verlag B.G. Teubner (1977)

9. Overhoff, H.M., Jan, U. von: Robustheitseigenschaften eines Algorithmus zur automatischen Vermessung des Femurkopfes in 3D-Ultraschallbildvolumina der Säuglingshüfte. In: Muche, R. et.al. (eds.), Medizinische Informatik, Biometrie und Epidemiologie: GMDS '97, MMV Medizin Verlag, München (1997) 106-110

10.Graf, R.: Sonographie der Säuglingshüfte und therapeutische Konsequenzen: ein Kompendium. 4th edition, Enke-Verlag Stuttgart (1993) 


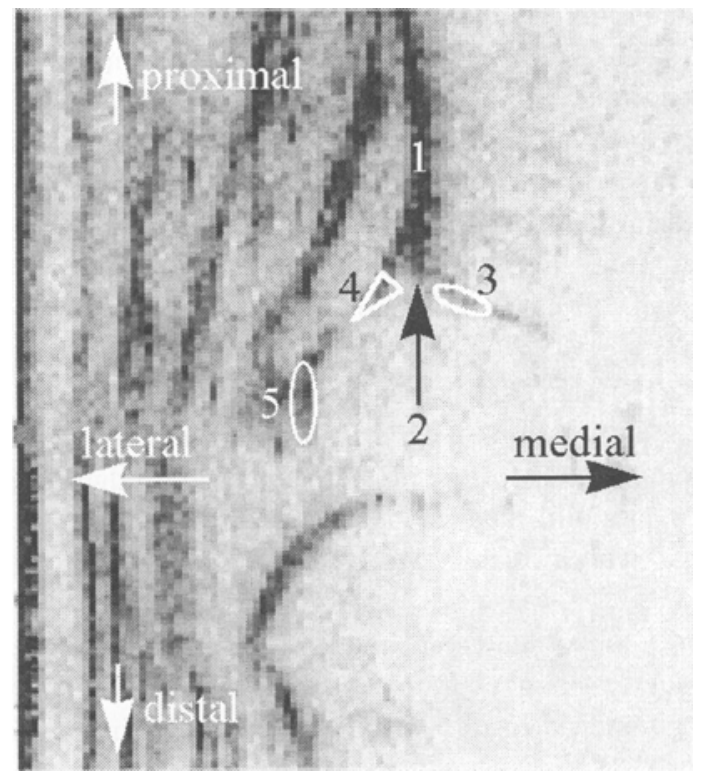

Fig. 1. Ultrasound image of the newborn's hip joint in Graf's standard plane (grey-scale inverted): anatomic orientation and landmarks according to Graf [3], [4]; numbers see text.

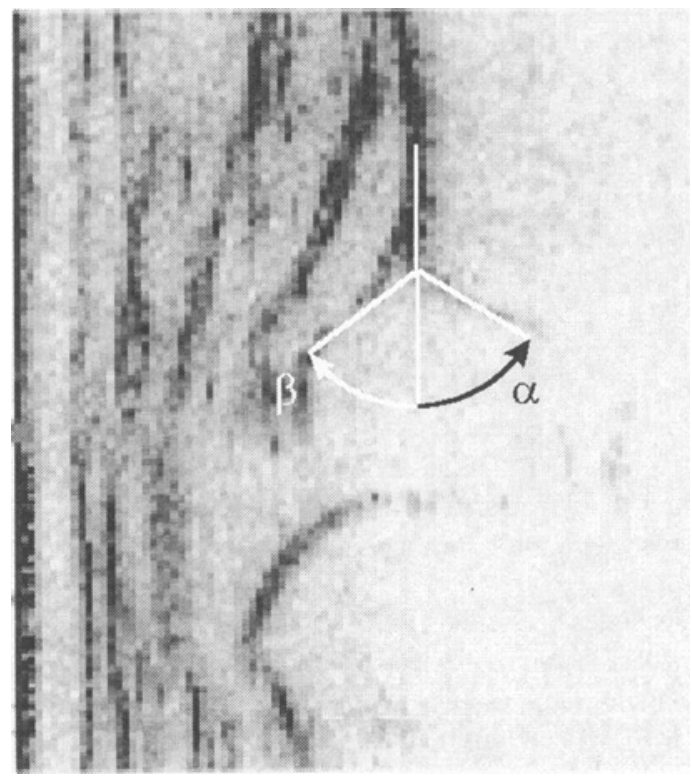

Fig. 2. Ultrasound image with diagnostic angles $\alpha$ and $\beta$. 


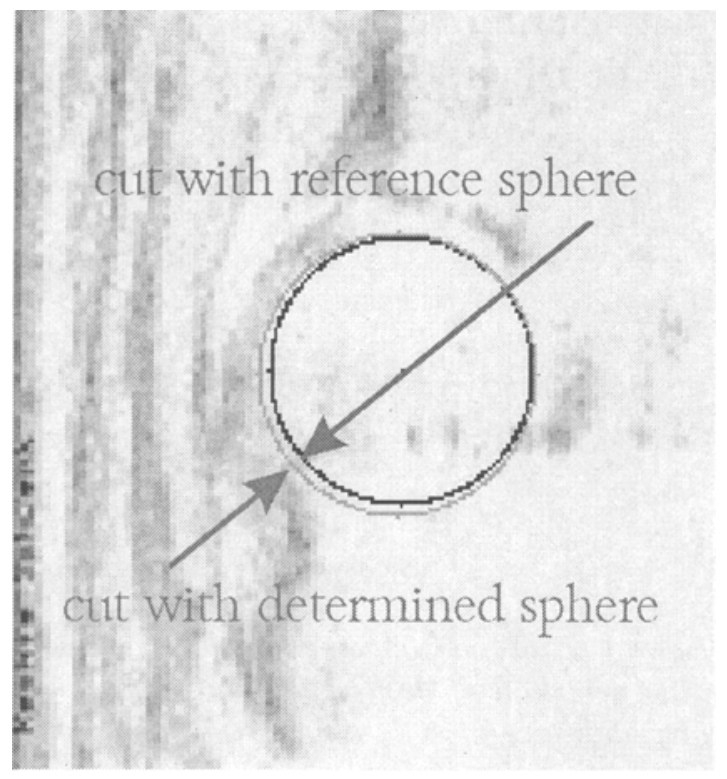

Fig. 3. Automatically determined femoral head approximating (dark grey) and reference sphere (black) intersecting an ultrasound image plane. The difference of the sphere diameters here is about $0.75 \mathrm{~mm}$.

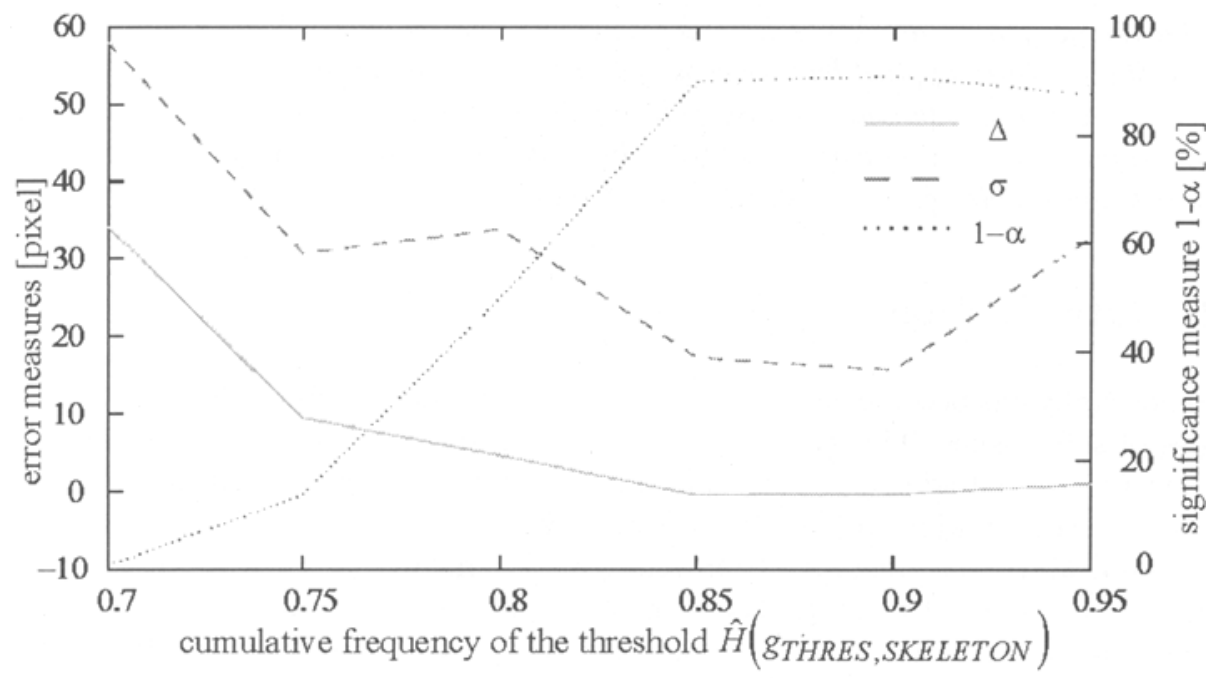

Fig. 4. Displacement of the $y$-co-ordinate of the most distal point of the iliac crest line. $\Delta$ : displacement error, $\sigma$ : standard deviation of $\Delta, \alpha$ : significance level 